Keio J. Med. 25: 101-110, 1976

\title{
MICROSURGERY IN DAILY PLASTIC SURGERY
}

\author{
TOYOMI FUJINO \\ Department of Plastic and Reconstructive Surgery, School of Medicine, \\ Keio University, 35 Shinanomachi, Shinjukuku, Tokyo, 160, Japan
}

(Received for publication June 24, 1976)

\begin{abstract}
Microsurgery is divided into 3 categories: microdissection, microvascular surgery and microneurorrhaphy. In plastic surgery, the latter two have been already practiced, but the microdissection technique is not popular and reported yet. This paper first reports the clinical application of the microdissection technique in the 50 common plastic surgical cases. The advantages to use the operating microscope with the lower magnifying power $(5-8 \times)$ were described in the representative cases; primary repair of laceration of the facial skin, syndactylia of the toe with a full-thickness skin graft, primary repair of the cleft lip, primary repair of the cleft palate, resection of the sebaceous cyst, hypospadias repair and removal of the subcutaneously buried suture.

In conclusion, the microdissection technique produces more atraumatic and refined surgical results than those by the conventional plastic surgical procedures.
\end{abstract}

In plastic surgery, microvascular surgery has been one of the topics in the recent literature. ${ }^{1}$ Yet many of us still think that it is a rather cumbersome procedure, requiring the special training and skill before clinical application. Success of microvascular transfer of a free flap is based on an atraumatic handling technique of the target tissues and vessels by microscopic magnification. This technique is a refined surgical principle and is applicable to our daily plastic surgical cases, as already practiced in the field of ENT, EYE and Brain surgeries. ${ }^{2-3}$ This paper first describes the clinical application of microsurgical technique in common plastic surgical cases and the table 1 shows a list of the operated cases number. The magnification of the operating microscope (Highbridge K280) is $5-8 \times$. (Fig. 1) 
Table 1

Total cases number treated by microdissection technique

\begin{tabular}{lrlll}
\hline \hline Facial tumors & 21 & Telecanthus & 1 \\
Laceration and scar revision & 14 & Polydactylia & 1 \\
Cleft palate & 3 & Syndactylia & 1 \\
Hypospadias & 3 & Alopecia & 1 \\
Status post-cheiloplasty & 3 & Septal deviation & \\
Cleft lip & 1 & & Total number & 50 \\
\cline { 3 - 4 } & & & & 1 \\
& & & &
\end{tabular}

\section{REPRESENTATIVE CASES REPORTS}

Case 1. Primary repair of laceration of the facial skin

A 44 year old male was referred to us immediately after an accident. Examination under the microscope revealed a half moon shaped and somewhat contaminated laceration, approximately $2 \mathrm{~cm}$ long, on the upper lateral eyelid on the left. The dirts around the wound were abraded by an edge of no, 11 blade. Minimal debridement of the wound edge and base was outlined and performed. Primary closure was done by 6-0 nylon interrupted sutures. All sutures were removed on day 5, when almost no inflammatory reaction or suture marks was noted. Suture line on day 7 was almost invisible (Fig. 2).

Comments: Thorough cleaning of the contaminated wound is often difficult under the naked eye, even under the recommended bright light. Microscopic examination of the wound demonstrates clearly an exact size and distribution of even the very small particles of the dirts. Therefore, it is easy to remove them mechanically unless the time of surgery is limited. As an exact determination of the damaged wound edge is often difficult under the naked eye examination, it is our routine to close the wound, especially of the face, primarily and do the secondary revision. Microsurgical debridement of the wound makes it possible to sacrifice only necessary yet minimal amount of the damaged tissue. Those two factors contribute to the minimal inflammatory reaction and better healing of the wound.

Desired tightness of the suture knot in order to avoid the suture marks is fundamental in surgery and owes to surgeon's skill. Our experiences under the microscope indicate that a just enough tightness of suturing tie to approximate the wound edges is desirable one and easily performed under the microscopic 
surgery.

We use either 6-0 or 7-0 nylon suture for skin closure and sometimes encounter the problems to remove them because of difficulty to catch an end of suture tail and insert a scissor between the narrow loop of the suture. Microscopic magnification smoothes the procedure without skin trauma nor leaving the remnant of the sutures.

Case 2. Syndactylia of the toe with a full tickness skin graft

A 9 month old girl revealed a syndactylia of her 3rd and 4 th toes on the left. Microscopic dissection was performed, preserving the digital nerve and vessels. Open wound was covered by a full thickness skin graft from the inguinal region. Under the microscope, a full-thickness skin graft was taken from the inguinal region by a sharp dissection technique. Three weeks postoperative finding showed a complete take of the grafted skin (Fig. 3).

Comments: A full-thickness skin graft is usually taken by either a sharp or blunt dissection technique, depending on a surgeon's choice. Even by an experienced surgeon, it is not rare to make a hole during a trimming-off procedure of an excessive fatty tissue after blunt dissection or a primary sharp dissection. This type of the trouble is avoided by the use of the microdissection, even by a sharp dissection technique. Yet, the most atraumatic full-thickness skin graft is obtained. Without mention, the obtained graft is immediately used, without any trimming-off procedure.

Case 3. Primary repair of the cleft lip

A 3 months old girl underwent a cheiloplasty along a Millard technique for a unilateral cleft lip on the left. One of the key points in the cheiloplasty is a production of a matched vermillion border. Under the microscope, the vermillion border was marked and sutured. The surgery was completed.

Comments: We see occasionally a mismatched vermillion border during the postoperative check. This could be avoided if one would mark the vermillion border by a methylene blue, as usually we do, and then suture it under the operating microscope. Total microsurgical cheiloplasty could be done but not necessary in order to save the surgical time.

Case 4. Primary repair of the cleft palate

A 1.5 year old boy underwent a palatoplasty along the push-back procedure 
for an incomplete cleft palate. Microsurgically, the great palatine neurovascular bundle was isolated and the levator velli palatine muscle was severed at the point over the hamulus process, followed by the suturing the clefted palate (Fig. 4).

Comments: The most important aim in the palatoplasty is a complete recovery of the speech. In recent trend, the distorted fibers of the levator velli palatine muscle is isolated from the hard palate and rearranged to a normal anatomical position. This is easily done under the microscope, even without injuring the nasal mucosa. Dissection between the great palatine neurovascular bundle and the retromolar region is difficult, yet we have to cut through the tight anchoring tissue in order to approximate the clefted palate in midline. Microsurgical dissection aids us greatly in preserving the lesser palatine neurovascular tissues.

Case 5. Resection of the sebaceous cyst

A 58 year old male presented a history of a tumor on the right cheek of 10 year duration. At surgery, the size of the tumor was $20 \times 15 \mathrm{~mm}$. An elliptical incision, $15 \mathrm{~mm}$ long, with a central linear incision including an efferent outlet, $9 \mathrm{~mm}$ long, was outlined. First, the sebaceous cystic content was curretted out through the central linear incision. Then, the sac of the cyst attached with the elliptical overlying skin was excised. The wound was closed with slightly tight knot. On day 4 , all sutures were removed, at which time a small erosion near the suture line at the center and suture marks were noted microscopically. On day 7, they were almost subsided (Fig. 5).

Comments : Removal of the sebaceous cyst by a minimal incision is imperative, especially on the face. One of the procedure is a currettage of the cystic content, followed by a secondary removal of the sac. Microsurgical currettage of the cystic content comfirms a complete removal of the sebum from the wound and makes it possible to remove the sac and close the wound primarily, without fear of recurrence.

A small erosion near the suture line at the center is usually ignored by the naked-eye examination. On reflecting back of the initial incision lines, one might notice a small hole at the central portion of the inner elliptical incision. This hole was not excised, and penetrated through a nylon suture, because during the microscopic dissection of the cyst, the hole was not connected with the cyst and then left intact. However, postoperative appearance of the skin erosion coincided 
with an indifferent outlet. This teaches us that any adjacent outlet near the main outlet has to be excised or should not be penetrated through the suture in order to get the better wound healing process. On reviewing the final outcome, this kind of the finding is rather exaggerated under the microscopic magnification, comparing to that under the naked eye, but it is important to obtain the better and refined surgical result.

Case 6. Hypospadias repair

A 3 year old boy underwent the reconstruction of a new urethra for a penile hypospadias. Parallel incisions were made along the ventral surface of the glans and penis. The skin flaps were raised laterally. The opposing skin edges of the glans and penis were united to form a new urethral tube by a two layer closure with 11-0 monofilament nylon interupted sutures. The lateral flaps were closed with 8-0 nylon sutures except for the portion of the glans where 11-0 nylon sutures were used. No catheter was inserted through the new urethra. Instead, suprapublic cystotomy was performed. On day 9 , all sutures were removed. All procedures were done under the microscope. On day 10 , urine was voided through the newly formed urethra. In two weeks, the patient was discharged from the hospital.

Comments: Numerous numbers of the reconstructive procedures for the hypospadias have been reported but everyone believes that the simpler is the better. Our method is a simple one and microdissection technique demonstrates an easy layer dissection, and less traumatic and hemorrhagic procedure. Therefore, this is even utilized for the secondary closure of the fistula.

Case 7. Removal of the subcutaneously buried sutures

A 24 year old female underwent a scar revision of the face due to a car accident. Six months later, she complained that she palpated a piece of a glass on the scar. Naked eye examination revealed a yellowish coated hard point on the scar. Microscopic examination showed a subcutaneously buried synthetic suture. This was removed by the microsurgical technique (Fig. 7).

Comments: Recently plastic surgeons tend to use the synthetic suture to approximate the subcutaneous layer in order to avoid the wound separation. Three to nine months after surgery, however, the synthetic suture tend to break through the overlying skin. Early discovery and removal are recommended. This is only possible under the microscopic maneuver, not under the naked-eye one. 


\section{REFERENCES}

1. Fujino, T.: Microvascular surgery in reconstructive plastic surgery. Keio J. Med. 23: $137-163,1974$

2. Holmgren, G.: Some experiences in surgery in otosclerosis. Acta Otolaryng. 5: $460-466,1923$

3. Perrit, R. A.: Recent advantages in corneal surgery. Trans. Amer. Acad. Ophth. Otolaryng. 54: 482, 1949

4. Yasargil, M. D.: Microsurgery applied to neurosurgery. pp. 220. George Thieme Verlag, Stuttgart, 1969 


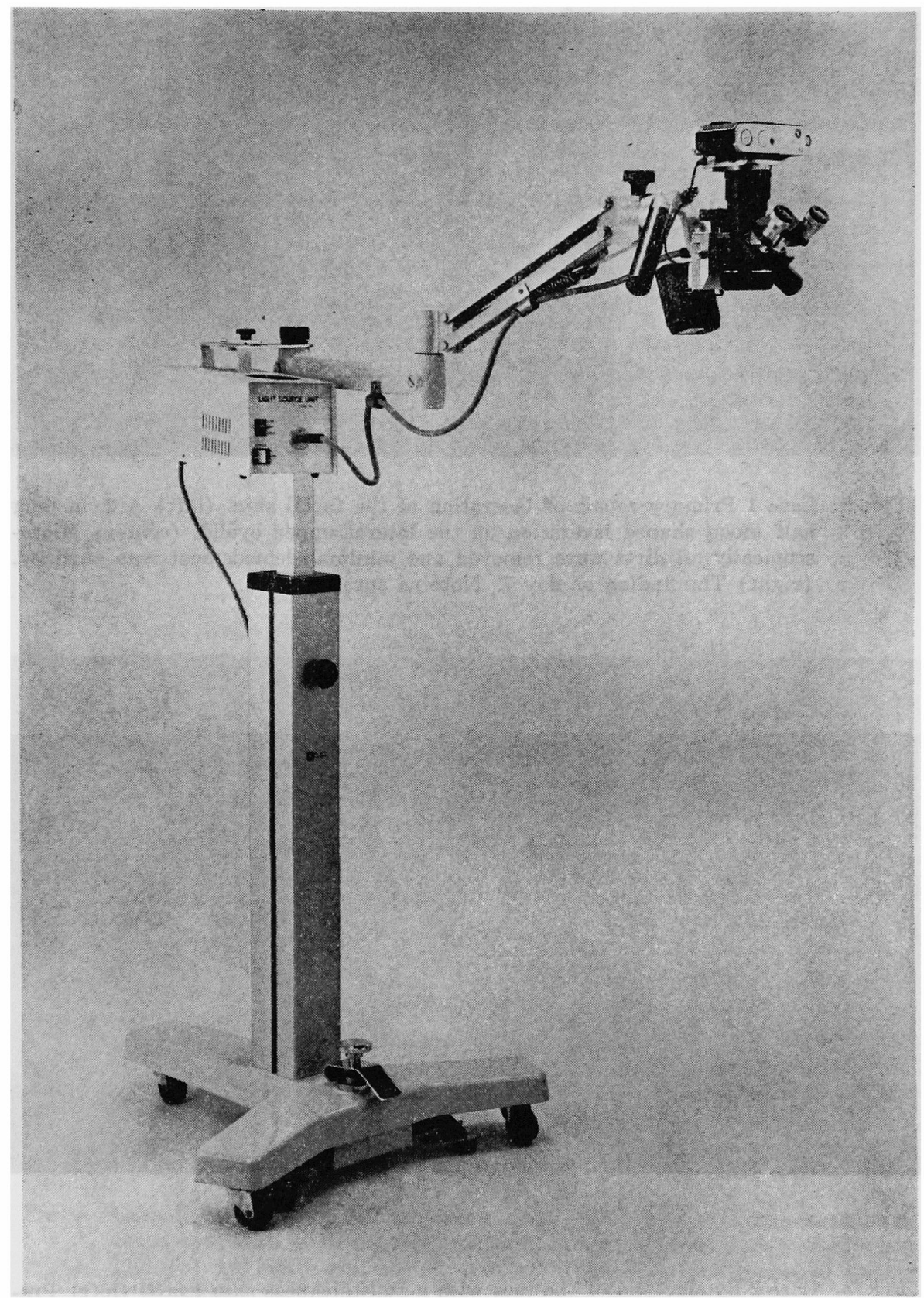

Fig. 1 Operating microscope (Highbridge type K-280) Magnifying power is $5-8 \times$.

(Highbridge Co. 1-132, Utsubo, Nishiku, Osaka, Japan 550) 


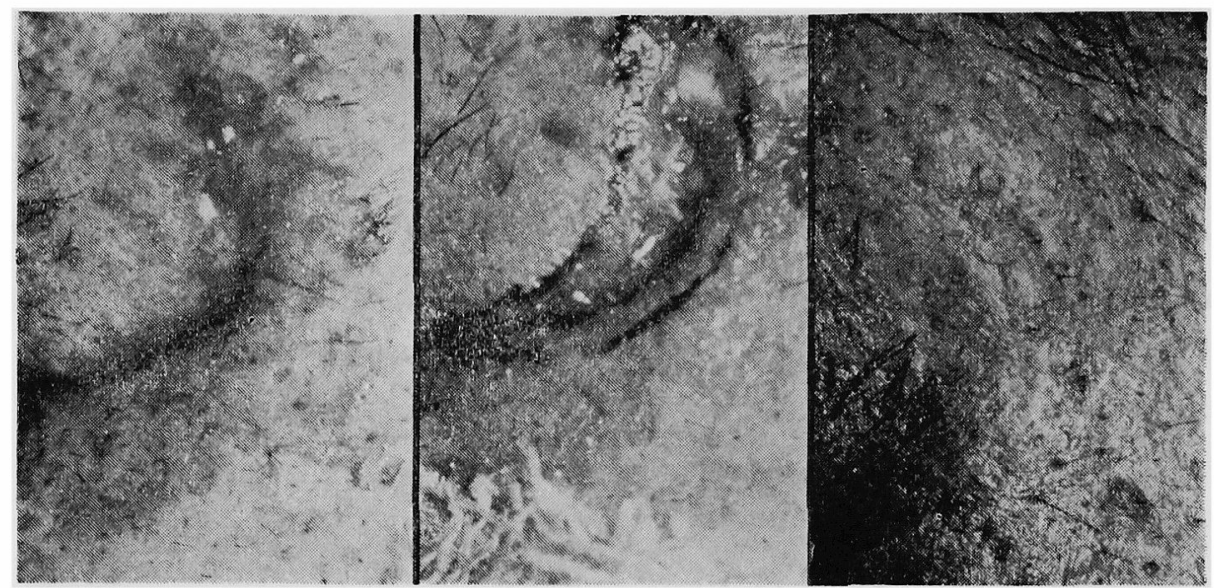

Fig. 2 Case 1 Primary repair of laceration of the facial skin. (left) A $2 \mathrm{~cm}$ long half moon shaped laceration on the lateral upper eyelid. (center) Microscopically all dirts were removed and minimal debridement was ourlined. (right) The finding on day 7 . Note no suture marks.

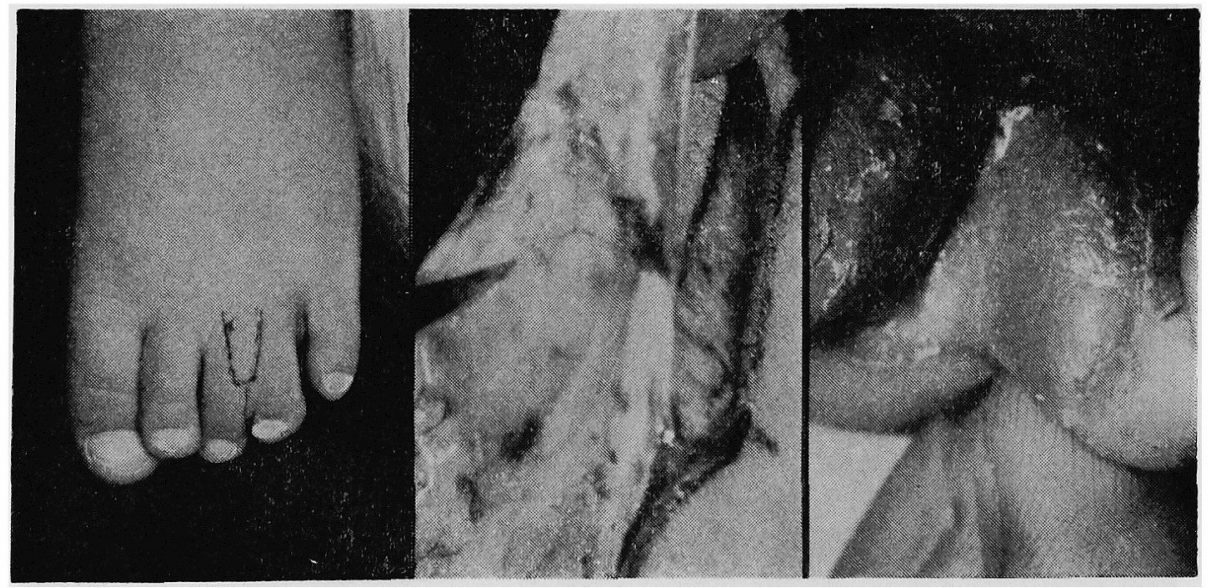

Fig. 3 Case 2 Syndactylia of the toes with a full-thickness skin graft. (left) Preoperative design. (center) Microsurgical sharp dissection of the fullthickness skin graft from the inguinal region. (right) Three weeks postoperative finding with a complete take of the grafted skin. 


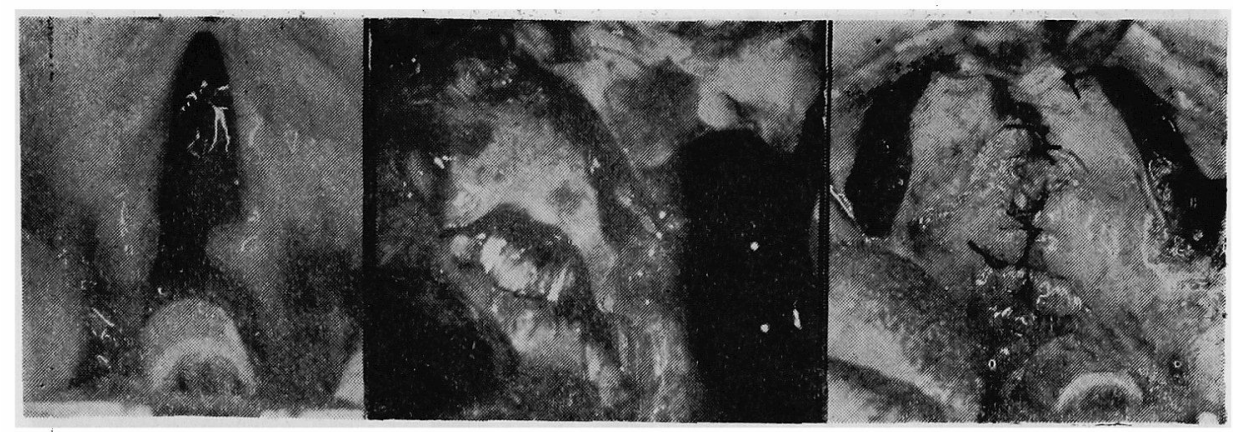

Fig. 4 Case 4 Primary repair of the celft palate. (left) Preoperative finding of the soft cleft palate. (center) The apponeurosis of the levator velli palatine muscle was clearly demonstrated and easily dissected out without : injuring the nasal mucosa under the microscope.

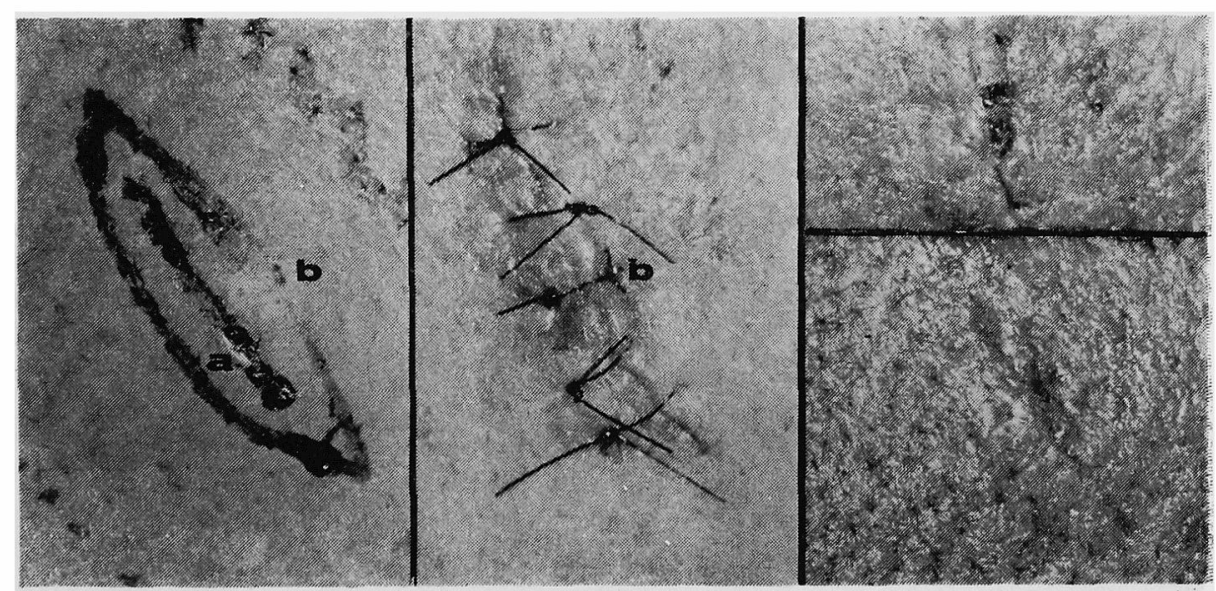

Fig. 5 Case 5 Resection of the sebaceous cyst. (left) A $20 \times 15 \mathrm{~mm}$ sized sebaceous cyst with a $15 \mathrm{~mm}$ long incision (a) an efferent outlet of the cyst and (b) an indifferent outlet. (center) Immediate postoperative finding with slightly tight suturing. The indifferent hole was penetrated through a suture. (right upper) A small skin erosion around the indifferent hole on day 4. (right lower) The finding with disappearing suture mark and erosion on day 7 . 


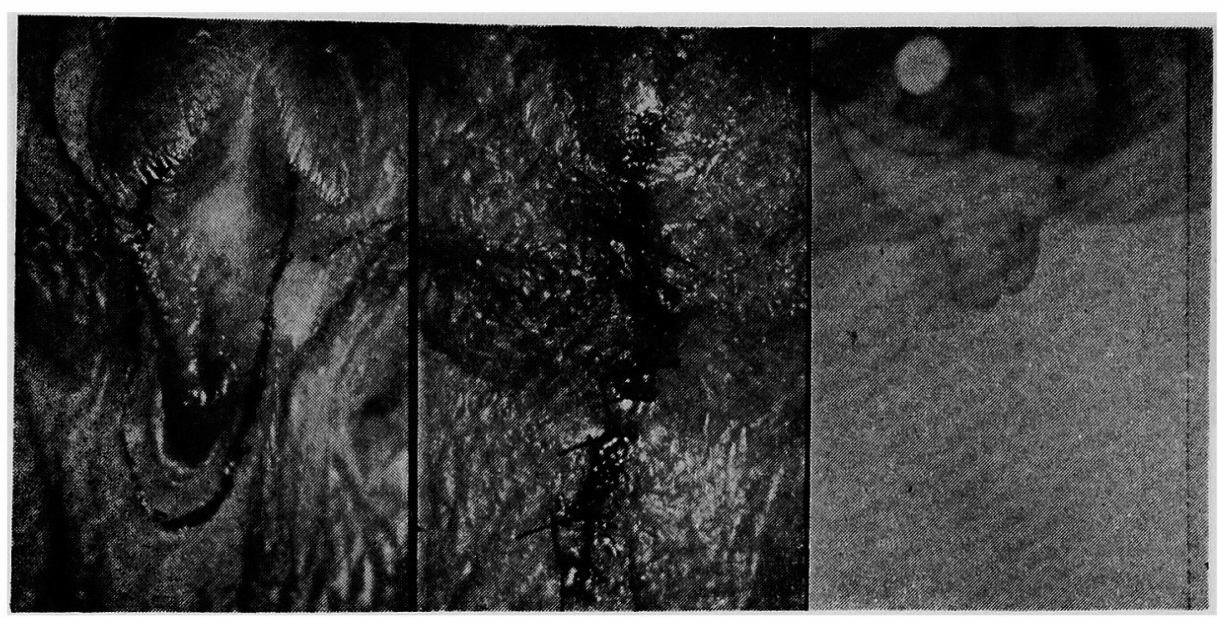

Fig. 6 Case 6 Hypospadias repair. (left) Design of the incision. (center) Skin was closed with 8-0 nylon interrupted suture except for the glans where 11-0 monofilament nylon suture was used. (right) Postoperative voiding.

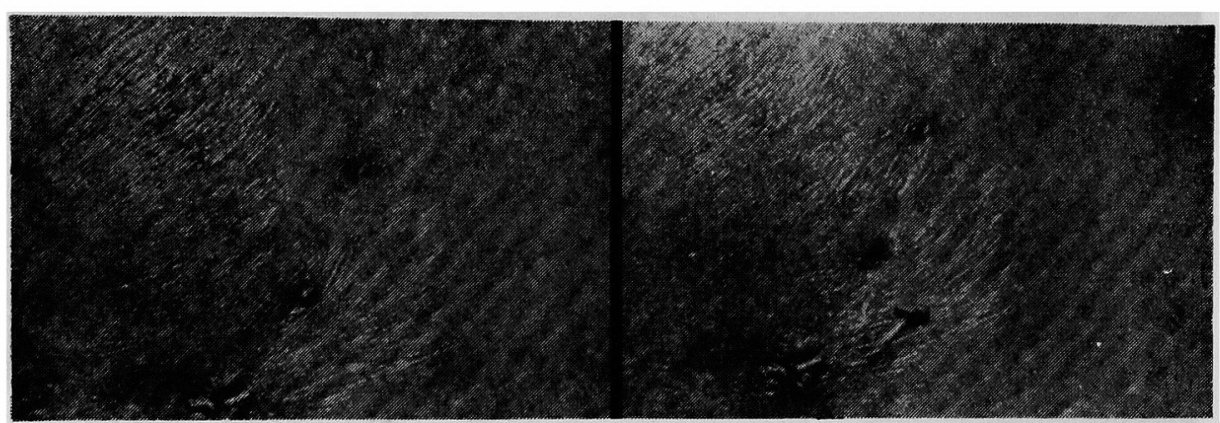

Fig. 7 Case 7 Removal of subcutaneously buried suture. (left) Microscopic finding of a subcutaneously buried suture (6-0 Tetlon suture). (right) After removal of the suture. 